\title{
Prefrontal Cortex Structure Predicts Training-Induced Improvements in Multitasking Performance
}

\author{
Ashika Verghese, ${ }^{1 *}$ ○K.G. Garner, ${ }^{1 *} \oplus^{\oplus}$ Jason B. Mattingley, ${ }^{1,2}$ and ${ }^{\circledR P a u l ~ E . ~ D u x ~}{ }^{1}$ \\ ${ }^{1}$ School of Psychology and ${ }^{2}$ Queensland Brain Institute, The University of Queensland, St. Lucia, Queensland 4072, Australia
}

The ability to perform multiple, concurrent tasks efficiently is a much-desired cognitive skill, but one that remains elusive due to the brain's inherent information-processing limitations. Multitasking performance can, however, be greatly improved through cognitive training (Van Selst et al., 1999, Dux et al., 2009). Previous studies have examined how patterns of brain activity change following training (for review, see Kelly and Garavan, 2005). Here, in a large-scale human behavioral and imaging study of 100 healthy adults, we tested whether multitasking training benefits, assessed using a standard dual-task paradigm, are associated with variability in brain structure. We found that the volume of the rostral part of the left dorsolateral prefrontal cortex (DLPFC) predicted an individual's response to training. Critically, this association was observed exclusively in a task-specific training group, and not in an active-training control group. Our findings reveal a link between DLPFC structure and an individual's propensity to gain from training on a task that taps the limits of cognitive control.

Key words: attention; individual differences; MRI; multitasking; training

\section{Significance Statement}

Cognitive "brain" training is a rapidly growing, multibillion dollar industry (Hayden, 2012) that has been touted as the panacea for a variety of disorders that result in cognitive decline. A key process targeted by such training is "cognitive control." Here, we combined an established cognitive control measure, multitasking ability, with structural brain imaging in a sample of 100 participants. Our goal was to determine whether individual differences in brain structure predict the extent to which people derive measurable benefits from a cognitive training regime. Ours is the first study to identify a structural brain marker - volume of left hemisphere dorsolateral prefrontal cortex - associated with the magnitude of multitasking performance benefits induced by training at an individual level.

\section{Introduction}

In today's fast-paced and increasingly digital world, the need to multitask, with the goal of getting more done in less time, has shifted from being chimerical to an everyday reality. Individuals are bombarded with new sensory information almost continually, all of which competes for attentional resources and neural

Received Sept. 11, 2015; revised Jan. 18, 2016; accepted Jan. 25, 2016.

Author contributions: A.V., K.G.G., J.B.M., and P.E.D. designed research; K.G.G. and P.E.D. performed research; A.V., K.G.G., and P.E.D. contributed unpublished reagents/analytic tools; A.V., K.G.G., and P.E.D. analyzed data; A.V., K.G.G., J.B.M., and P.E.D. wrote the paper.

This work was supported by Australian Research Council (ARC) Discovery Grant DP140100266, ARC SRI Science of Learning Research Centre Grant SR120300015, and ARC Centre of Excellence for Integrative Brain Function Grant CE140100007. P.E.D. was supported by ARC Future Fellowship FT120100033 and a University of Queensland Foundation Research Excellence Award. J.B.M. was supported by ARC Australian Laureate Fellowship FL110100103. We thank Luke Hearne and Amy Taylor for assistance with participant recruitment and data collection.

${ }^{*}$ A.V. and K.G.G. contributed equally to this work.

The authors declare no competing financial interests.

Correspondence should be addressed to Ashika Verghese or Paul E. Dux at the above address. E-mail: a.verghese@uq.ed.au or paul.e.dux@gmail.com.

DOI:10.1523/JNEUROSCI.3410-15.2016

Copyright $\odot 2016$ the authors $\quad 0270-6474 / 16 / 362638-08 \$ 15.00 / 0$ representation (Desimone and Duncan, 1995). Despite continued exposure to this complex sensory world, humans are notoriously poor at undertaking multiple tasks concurrently, even when component tasks require simple sensorimotor translation, such as mapping a single stimulus (e.g., a red circle) to a motor response (e.g., a single-finger button press; Pashler, 1994). Fortunately, multitasking performance can be markedly improved with extensive training (Van Selst et al., 1999; Strobach et al., 2013). Although the mechanisms underlying such training effects are debated (Ruthruff et al., 2006; Kamienkowski et al., 2011; Strobach et al., 2013), along with issues concerning the extent to which training benefits transfer to new tasks (Owen et al., 2010; Jaeggi et al., 2011), several functional imaging studies have shown that reductions in neural activity in the dorsolateral prefrontal cortex (DLPFC), anterior cingulate cortex, and posterior lateral prefrontal cortex track training-induced performance gains (Chein and Schneider, 2005; Erickson et al., 2007; Dux et al., 2009). To date, however, no study has examined the corresponding structural correlates that predict the extent to which an individual is likely to respond to training. Here we examined whether 
A

\begin{tabular}{|c|c|c|c|}
\hline$\frac{\text { Familiarization }}{\text { Friday }}$ & $\frac{\text { Pre-training MRI }}{\text { Monday }}$ & $\begin{array}{l}\text { Training sessions } \\
\text { Tuesday,Wednesday,Thursday }\end{array}$ & $\frac{\text { Post-training MRI }}{\text { Friday }}$ \\
\hline $\begin{array}{c}\text { Multitasking } \\
\text { paradigm } \\
\text { (with feedback) }\end{array}$ & $\begin{array}{c}\text { Multitasking } \\
\text { paradigm } \\
\text { (no feedback) }\end{array}$ & $\begin{array}{l}\text { Relevant Training Group } \\
\text { Multitasking paradigm } \\
\text { ( } 3000 \text { trials) } \\
\text { OR } \\
\text { Irrelevant Training Group } \\
\text { Visual search paradigm } \\
\text { (3000 trials) }\end{array}$ & $\begin{array}{l}\text { Multitasking } \\
\text { paradigm } \\
\text { (no feedback) }\end{array}$ \\
\hline
\end{tabular}

B

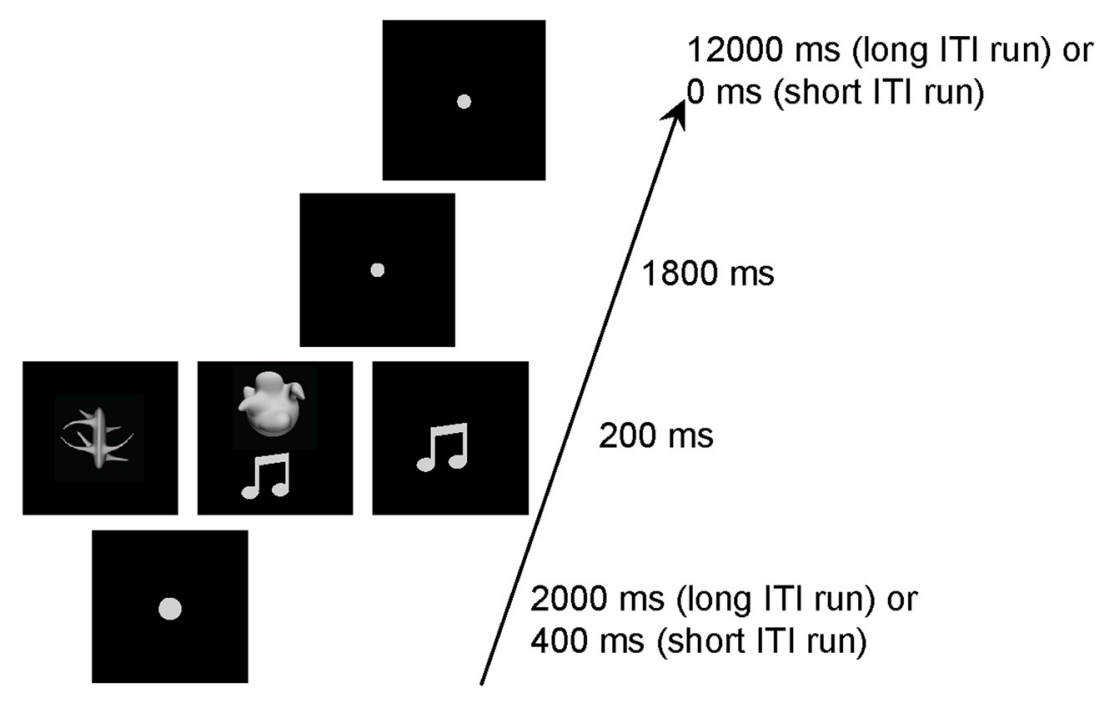

C

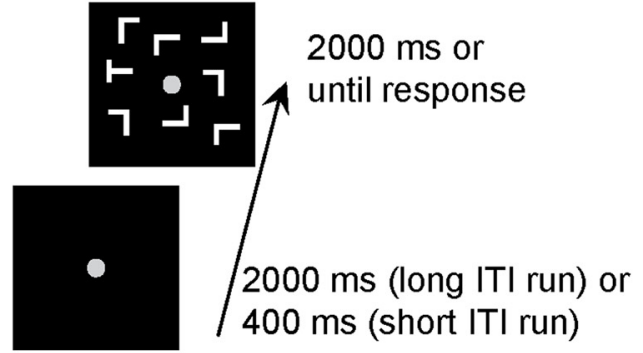

Figure 1. Testing protocol and behavioral paradigms. $\boldsymbol{A}$, Overview of the testing protocol for each participant. Familiarization sessions were run on Friday afternoon in the week before testing. MRI test sessions were conducted on Monday and Friday at the same time for each participant. Three days of training sessions were run in the interim period (Tuesday, Wednesday, Thursday). $\boldsymbol{B}$, Example display sequence for the multitasking paradigm. The task comprised three conditions: single task visual manual, in which participants made a manual response to one of two shapes (a "spikey" or a "smoothy" shape); single task auditory manual, in which participants responded manually to an auditory tone (two easily discernible, complex tones); and multitask, in which both single tasks were presented concurrently and each required a separate response. C, Visual search paradigm performed by the irrelevant training group. Participants judged the orientation of a letter "T" target (rotated by $90^{\circ}$ clockwise or counterclockwise) presented among 7 (small set size), 11 (medium set size), or 15 (large set size) rotated letter "L" distractors. This is a classic inefficient search paradigm.

improvements in multitasking performance via cognitive training are linked to the structure of specific brain areas.

Surface-based morphometry (SBM; Fischl and Dale, 2000) offers an effective tool for examining individual differences in brain structure by providing measurements for several key neuroanatomical properties, including cortical thickness, cortical surface area, and gray matter volume, all of which are potentially independent (Panizzon et al., 2009; Winkler et al., 2010). Here, we used SBM to determine whether regional cortical volume (the product of surface area and thickness) predicts an individual's response to multitasking training.

We tested two groups of participants. One group $(n=50)$ trained on a "task relevant" protocol (relevant training group; $n=50$ ), whereas the other trained on a "task irrelevant" regimen as a control (irrelevant training group; $n=50$ ). As depicted in Figure $1 A$, all participants underwent six testing sessions over the course of a week: a familiarization session and two magnetic resonance imaging (MRI) sessions (before and after training) that were interspersed with training sessions conducted outside the scanner across $3 \mathrm{~d}$. In the pretraining and the posttraining scanner sessions, participants performed single- and multitask trials to assess their multitasking performance capacity, which reflects their ability to coordinate and execute multiple tasks concurrently. To anticipate our findings, the relevant training group showed greater behavioral training benefits than the irrelevant training group. Critically, for the relevant training group only, there was a significant relationship between cortical volume in the left rostral middle frontal region and multitasking training improvements across individuals.

\section{Materials and Methods}

Participants

In total, 111 participants were tested in this study. Of these, 11 participants had to be excluded: 6 due to head motion $>5 \mathrm{~mm} /$ degree in any translational direction or rotation, 2 due to a failure of the sound equipment in one of the sessions, 1 for responding incorrectly to the sound task across all six runs, 1 due to technical errors in the first training session, and 1 withdrew from the study midway through participation. The re- 
maining 100 participants were randomly allocated into one of the two groups: relevant training (mean age in years $\pm \mathrm{SD}, 24.3 \pm 6.2 ; n=50,15$ males, 3 left-handers) and irrelevant training (mean age in years \pm SD, $24.6 \pm 5.5 ; n=50,11$ males, 4 left-handers). All participants provided informed written consent in accordance with The University of Queensland Human Research Ethics Committee. Data were collected as part of a previous investigation (Garner and Dux, 2015) and were analyzed here for the purposes of exploring the structural basis of individual differences in multitasking training outcomes.

\section{Behavioral sessions}

The experiment was programmed using Matlab R2010a (MathWorks) and the Psychophysics Toolbox (version 3.0.9 extension; Brainard, 1997; Pelli, 1997). The familiarization and behavioral training sessions were conducted with a 21 inch Sony Trinitron CRT monitor and a Macintosh $2.5 \mathrm{GHz}$ MacMini computer. The behavioral test sessions were run on a MacBook Pro laptop and presented on a rear projection screen set at distance of $90 \mathrm{~cm}$.

\section{Multitasking paradigm}

Familiarization session. On each trial, participants were presented with either a single- or multitask condition. As shown in Figure $1 \mathrm{~A}$, in the single-task trials, participants performed either a visual shape discrimination task (white smooth-edged vs spikey-edged shape subtending $6^{\circ}$ of visual angle against a black background) or an auditory complex-tone discrimination task (two easily discernible complex tones, as used in previous work; Dux et al., 2006). For the multitask condition, both the shape and the sound were presented simultaneously. Participants responded to visual stimuli using either the middle or the index finger of either the left or the right hand (task/hand assignment counterbalanced across participants). The other hand was used for responding to the auditory stimuli in a similar manner. Participants were instructed to respond as quickly and accurately as possible to both tasks and could freely respond to the stimuli in any order.

For the initial familiarization session, participants completed two runs of 18 trials each (equal number of trials for shape single task, sound single task and multitask). In the first run, referred to as the short intertrial interval (ITI) run, trials began with a fixation dot $\left(0.5^{\circ}\right.$ visual angle) presented for $400 \mathrm{~ms}$, which was followed by the stimulus or stimuli for $200 \mathrm{~ms}$. Finally, a response screen containing a smaller fixation dot $\left(0.25^{\circ}\right)$ appeared for $1800 \mathrm{~ms}$. Accuracy feedback was provided after each trial, and participants repeated the run until they achieved five of six trials correct for each trial type.

The second run used a longer ITI to familiarize participants with the timings used in the MRI test sequence. Here, the alerting fixation was presented for $2000 \mathrm{~ms}$, followed by the stimulus presentation (200 ms), the response period (smaller fixation dot for $1800 \mathrm{~ms}$ ), and finally an interval of 12,000 ms, during which the smaller fixation dot remained on screen. Participants received feedback at the end of each trial.

MRI sessions. The pretraining and posttraining MRI sessions were identical to the long run of the familiarization session. The only difference was that no feedback was provided in these trials. Participants completed six runs in the scanner, with 18 trials per run (six of each trial type, pseudorandomly ordered for each run), for a total of 108 trials for the session.

\section{Training sessions}

Participants were randomly assigned to either a relevant training group $(n=50)$ or an irrelevant training group $(n=50)$. The relevant training group trained on the same multitasking protocol as used in the pretraining and posttraining sessions. On each day, participants completed 56 blocks of 18 trials, resulting in 3024 training trials overall. To allow for a high number of training trials, while retaining familiarity with the task timings, between two and four blocks used a long ITI (2000 ms alerting cue and 12,000 ms interval following the response), and the remaining blocks used a short ITI (400 ms alerting cue without any interval following the response period).

The irrelevant training group, by contrast, trained on a visual search task in which participants reported the orientation of a white rotated letter $\mathrm{T}\left(\sim 1^{\circ}\right.$ size, oriented left or right) presented among a variable number $(7,11$, or 15$)$ of rotated Ls against a black background (Fig. 1B). Trials commenced with either a 2000 or $400 \mathrm{~ms}$ fixation screen and were then followed by the search display that remained on screen for $2000 \mathrm{~ms}$ or until response. As with the relevant training group, participants completed 3024 trials in all (1008 trials for each set size).

For both groups, performance feedback was provided. Specifically, a point system was used to keep participants motivated. If participants met their response time (RT) target (initial target of $2 \mathrm{~s}$ ) for over $90 \%$ of trials and maintained $90 \%$ accuracy or greater, they were awarded two points. A new RT target was calculated using the 75th percentile of the RTs from the previous eight blocks. If participants did not improve upon their RT target for over $90 \%$ trials, but still maintained $>90 \%$ accuracy, one point was awarded, while the RT target remained the same. If neither the RT target nor the target accuracy criterion were met, no points were awarded and the RT target did not change. At the end of every eight blocks, a visual feedback message was provided summarizing the mean RT and mean accuracy for the previous eight blocks (collapsed across the two singletasks for the relevant training group and over the three set sizes for the irrelevant training group) as well as the number of points scored and the RT target for the subsequent eight blocks.

\section{MRI sessions}

Images were acquired using a 3T Siemens Trio MRI scanner. Participants lay supine in the scanner and viewed the visual display via rear projection onto a mirror mounted on a 12-channel head coil. A high-resolution T1-weighted structural image was collected from each participant both before and after the training sessions (i.e., on Monday and Friday; repetition time, $1.9 \mathrm{~s}$; echo time, $2.32 \mathrm{~ms}$; flip angle, $9^{\circ}$; field of view, $192 \times$ $230 \times 256 \mathrm{~mm}$; resolution, $1 \mathrm{~mm}^{3}$ ).

\section{MRI data analysis}

Pretraining T1 scans for 100 participants were processed using FreeSurfer (Fischl et al., 1999). This automated reconstruction procedure performs segmentation and reconstruction of the pial surface (Fischl and Dale, 2000). The volume measures were smoothed using a Gaussian kernel with a full width at half maximum of $10 \mathrm{~mm}$. A multiple regression was performed to identify cortical regions that showed correlations with multitasking performance changes from pretraining to posttraining. The gender and age of each participant were included in the design matrix as nuisance parameters, and their effects were regressed out (Escorial et al., 2015). Clusterwise correction for multiple comparisons was performed using Monte Carlo simulation, with 10,000 simulations for estimating the probability of forming a cluster of a given size by chance when clusters were obtained by a vertexwise threshold of $p<0.01$. Clusterwise $p$ values of 0.05 were used as the minimum criterion for significant clusters. For regions that showed significance (after Monte Carlo correction) in the group analysis, labels were drawn on the average surface and then mapped onto the individual participant. The relevant anatomical measures (volume, surface area, and thickness) were then extracted for each participant. To verify our regression results, we also conducted crossvalidation checks using split-half (Engvig et al., 2010) and leave-one-out strategies (Martens and Dardenne, 1998).

\section{Results}

An individual's multitasking performance was quantified as the reduction in $\mathrm{RT}$ for the multitasking trials compared with the single-task trials ([RT auditory stimulus multitask trials - RT auditory stimulus single-task trials] + [RT visual stimulus multitask trials - RT visual stimulus single-task trials]). Analyses of the behavioral data revealed that the relevant training group showed a significantly larger training benefit (i.e., a greater reduction in RT costs from pretraining to posttraining) than the irrelevant training group [repeated measures ANOVA, 2 (sessions) $\times$ 2 (groups) interaction effect, $F_{(1,98)}=31.12, p<0.0001$; Figure $2 A]$. An ANCOVA with pretraining performance as the covariate further corroborated that the posttraining multitasking performance improvement was significantly greater for the relevant training group than for the irrelevant training group, 
A

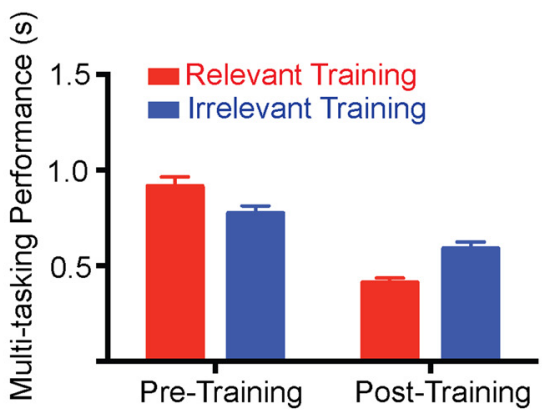

C

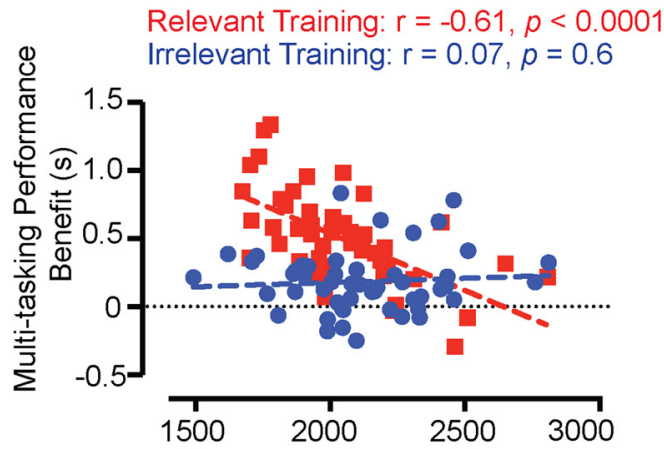

Left rostral DLPFC volume $\left(\mathrm{mm}^{3}\right)$

Figure 2. Results. $A$, Behavioral data. Improvements in multitasking performance from pretraining to posttraining sessions were greater for the relevant training group than for the irrelevant training group $(p<0.0001$; error bars represent SEM). $\boldsymbol{B}$, Whole-brain structural analysis. The cortical surface $p$ maps shows the left rostral DLPFC region for the relevant training group, whose volume (determined from pretraining $\mathrm{T} 1 \mathrm{scans}$ ) reliably predicted training-induced multitasking improvements (premultitasking cost minus postmultitasking cost). Clusters were thresholded at $p<0.01$ (corrected for multiple comparisons) across all participants in the group. $C$, Region of interest correlation. The pattern of correlation between the volumes of the significant DLPFC cluster in the two groups and multitasking performance benefit is depicted. Unlike the relevant training group (red symbols), the irrelevant training group (blue symbols) did not show a correlation between behavior and left rostral DLPFC volume.

A

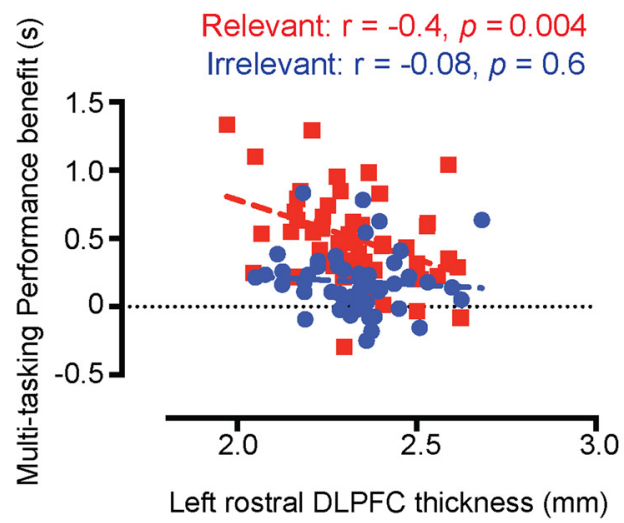

B

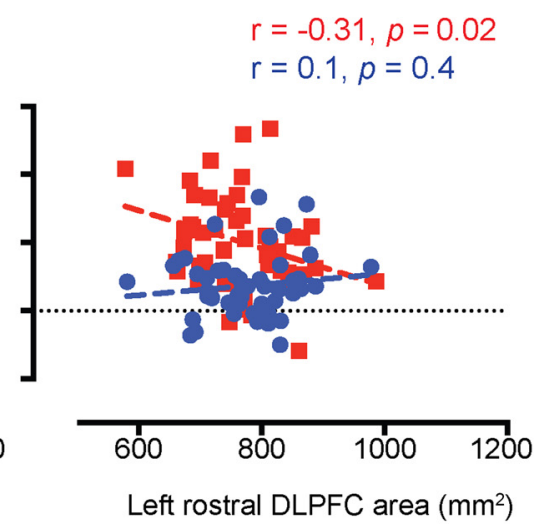

Figure 3. Correlations between multitasking performance benefit and cortical thickness and area. $\boldsymbol{A}$, Significant correlation is observed between left rostral DLPFC thickness and multitasking performance improvements in the relevant training group (red symbols) but not in the irrelevant training group (blue symbols). $\boldsymbol{B}$, Shows a similar pattern of results for the relationship between left rostral DLPFC area and multitasking performance benefit.

even after adjusting for the covariate $\left(F_{(1,97)}=32.9, p<\right.$ 0.0001 ). This confirms the efficacy of the experiment design by highlighting the role of the irrelevant training group as an active control.
SBM of the high-resolution structural MRI scans was used to examine whether variability in multitasking training benefits for the two groups (relevant vs irrelevant) could be predicted based on pretraining cortical volume measures. Training-induced multitasking performance benefits were defined as the reduction in multitasking performance costs from before to after training (pretraining multitasking performance minus posttraining multitasking performance). Multiple regression analysis comparing the two groups (relevant training or irrelevant training) revealed differential clusters in the precuneus, insula, lateral-occipital region, and left rostral middle frontal region. These regions showed stronger negative correlations between multitasking performance benefit and regional volume for the relevant training group relative to the irrelevant training group $(p<0.05)$. Further examination of the nature of these differences for each group independently, however, revealed that three of these clusters- the precuneus, insula and lateral-occipital region-were driven by positive correlations between multitasking performance benefit and cortical volume in the irrelevant training group (i.e., in these three regions, larger volumes correlated with improved multitasking performance benefit; precuneus, $r=0.58, p<0.0001$; insula, $r=0.49, p=$ 0.0003; lateral occipital, $r=0.63, p<$ 0.0001 , two-tailed, whole-brain analyses; Monte Carlo clusterwise correction for multiple comparisons). As the current design did not include a visual search task in the test session, we cannot easily explain which of several factors, such as task novelty or motivation, contributed toward the performance improvements observed in this group. On the other hand, as shown in Figure $2 B$, the relevant training group showed a significant correlation between cortical volume in the left rostral, middle frontal region [labels are from the atlas of Desikan et al. (2006)] and multitasking training improvements across individuals $(r=-0.473, p=0.0005$, twotailed, whole-brain analyses; Monte Carlo clusterwise correction for multiple comparisons). This region coincides with the rostral portion of the DLPFC (peak voxel; $x=-27, y=45, z=12$; labeled as BA46 in MNI space). Of note, the identical prefrontal cluster was obtained even after controlling for individual pretraining multitasking performance using a partial correlation approach $(r=0.3, p=0.01$, corrected for multiple comparisons). The result indicates that a lower cortical volume in left DLPFC is associated with a greater response to multitasking 
training. Similar patterns of correlation were also obtained using the raw volume, area, and thickness measures extracted from within the DLPFC region, providing further confirmation of our finding (volume, $r=-0.61$; thickness, $r=$ -0.4 ; area, $r=-0.31$; all $p$ values $<0.05$; Pearson's correlations for the region of interest; Figs. 2C, 3). Importantly, whole-brain analysis using the posttraining structural scans identified an identical left DLPFC cluster, thus replicating the results from the pretraining scans.

We also conducted two independent cross-validation analyses to verify our findings. First, we adopted a split-half strategy as used in other SBM studies (Engvig et al., 2010; Righart et al., 2013). The data set was first ordered according to group (relevant training or irrelevant training), gender, multitasking performance benefit, and age. Each group was then partitioned into two equally sized subgroups such that every alternate case was allotted to "Split 1" and the rest to "Split 2" (see Engvig et al., 2010). This yielded uniformly matched subsets. Independent, whole-brain general linear models were then run on all four split groups. This analysis contrasted relevant training Split 1 with irrelevant training Split 1, and relevant training Split 2 with irrelevant training Split 2 (for each subgroup, $n=$ 25). We also conducted similar split-half analyses using just the region-of-interest volumes (i.e., left rostral prefrontal volume). The results, depicted in Figure 4, demonstrate that left DLPFC volume significantly predicts the observed multitasking training benefit even with reduced sampling.

We also cross-validated the efficacy of our model predictors using the caret package in $\mathrm{R}$ (https://cran.r-project.org/web/ packages/caret/index.html). For this analysis, we used a leave-one-out cross-validation procedure, which is valid for small data sets, $n=40$ to 120 (Martens and Dardenne, 1998), to investigate whether the group allocation (relevant training group or irrelevant training group) could be determined on the basis of the following predictors: left rostral prefrontal volume, multitasking performance benefit, gender, and age. Under this cross-validation scheme, for each iteration, data were partitioned into training and test sets by allocating one participant to the test set and the remaining participants $(n=99)$ to the training set. The training set was then used to predict the outcome (group membership) using a gradient booster machine that autotunes parameters to obtain the best-fitting model. The resultant trained classifier was then used to predict the group membership of the excluded test participant. This process was repeated until each participant was left out once and a final accuracy measure [overall model classification accuracy based on the receiver$2, n=25$ per group).
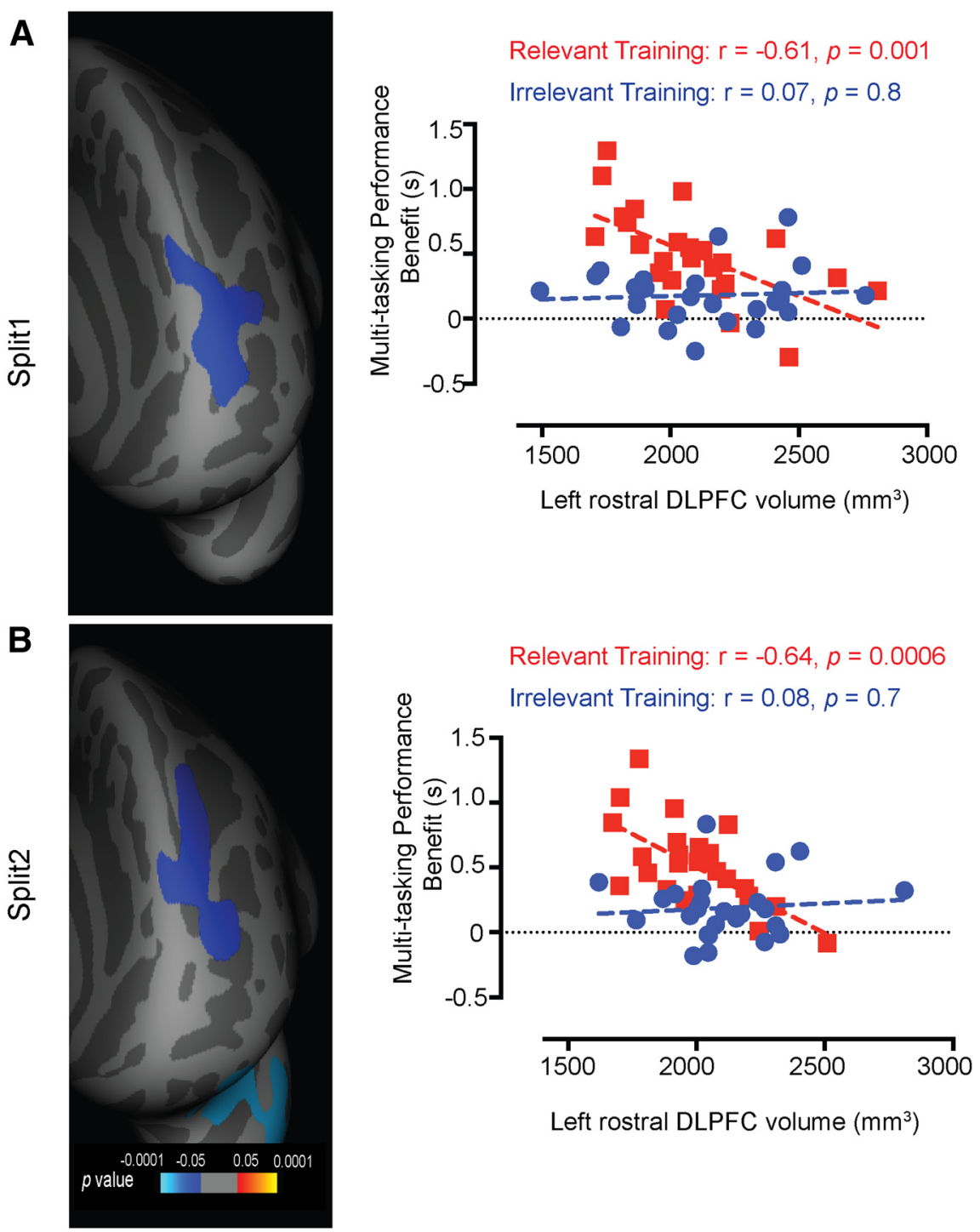

Left rostral DLPFC volume $\left(\mathrm{mm}^{3}\right)$

Figure 4. Split-half cross-validation. $\boldsymbol{A}$, The left panel depicts $p$ maps of the differences in the correlations observed between volume and multitasking performance benefit for the relevant training and the irrelevant training subgroups (Split 1), estimated by a general linear model analysis (corrected for multiple comparisons) using the split-half approach. The correlations in the prefrontal region were significantly stronger for the relevant training subgroup than for the irrelevant training subgroup. The $p$ value maps are thresholded at $p<0.05$ (Monte Carlo correction for multiple comparisons). The right panel shows multitasking 年 with a smaller subset (Split 1, $n=25$ per group). $\boldsymbol{B}$, The left panel shows a similar pattern of clustering using the Split 2 subgroups of the relevant training and the irrelevant training cohorts. The right panel also demonstrates that only the relevant training group shows a relationship between multitasking performance benefit and left rostral DLPFC volume even with reduced sampling (Split

operating characteristic (ROC) curve metrics] was obtained. The ROC curve is a function of the true positive rate (sensitivity) plotted against the false positive rate ( 1 minus specificity) at various cutoff thresholds and is used to determine the threshold with the best classification percentage. Our model provided an overall classification accuracy of 0.94 (95\% CI of 0.874 to $0.9777 ; p=2 \mathrm{e}-16)$. Sensitivity (i.e., accurate classification of the relevant training group) was 0.92 , whereas specificity (i.e., accurate classification of the irrelevant training group) was 0.96 . The best predictors ranked according to their relative influence in the model were multitasking performance benefit (50\%), left prefrontal volume (39\%), age (10\%), and gender (1\%). Together, the results from our validation procedures corrob- 
orate the robustness of our findings and show that the relationship between multitasking performance benefit and left prefrontal volume is a strong determinant of group (relevant training vs irrelevant training).

For the irrelevant training group, none of the prefrontal regions showed any significant correlation with training-induced multitasking performance benefits. This was verified by extracting volumes for the same left rostral DLPFC region in this group $(r=0.07, p=0.6$, Fig. 2C). A Fisher's $z$ test confirmed that the correlation coefficient between left rostral DLPFC volume and multitasking training benefit was significantly greater for the relevant training group than for the irrelevant training group $(z=3.1, p=0.004$, two-tailed). Similar patterns of significance were obtained using the thickness and area measures (area, $z=-2.04, p=0.04$; thickness, $z=-2.43, p=0.02$; two-tailed, Fisher's $z$ test).

In a control analysis, we verified that the observed correlations were specifically due to multitasking training and not to unidentified artifacts in the testing environment or scanner. We calculated multitasking performance differences from Day 1 to Day 3 of training to obtain a measure of multitasking performance outside the scanner. Whole-brain analyses for correlations between volume and multitasking performance benefit during the training phase (three participants who did not show a gain in multitasking performance were excluded) revealed a cluster in the prefrontal cortex $(r=-0.5, p=0.0001$, two-tailed) that overlapped with our results from the test sessions. Region-of-interest analyses also showed that the relevant training group demonstrated significant correlations between left rostral DLPFC volume and multitasking performance change in the training phase $(r=-0.32, p=0.02)$. By contrast, the irrelevant training group did not show any significant correlations between left rostral DLPFC volume and search training change across any of the three set sizes (all $p$ values $>0.05$; Fisher's $z$ test; Set $8, z=1.89, p<$ 0.05 ; Set 12, $z=1.75, p=0.07$; Set 16, $z=2.39, p<0.05$ ).

We next probed whether this anatomical training marker reflected a general training improvement for both single and multiple tasks or was specific to the multitasking condition. Whole-brain analysis comparing volume and task improvement on the multitask conditions only (pretraining multitask trial RT minus posttraining multitask trial RT) again revealed a significant left DLPFC cluster $(r=-0.4$, corrected $p=0.003$; Fig. 5$)$ in the relevant training group. There were no significant clusters for the same comparison conducted on the single-task data $(p>$ 0.05 , corrected). Critically, the two correlations differed significantly from each other ( $p<0.001$, uncorrected).

Finally, the present results do not appear to reflect baseline individual differences in multitasking performance or short-term plasticity. Whole-brain analysis in the relevant training group revealed no significant clusters for correlations between pretraining multitasking performance (RT multitask trials minus RT single-task trials) and cortical volume ( $p>0.05$, corrected), suggesting that the correlations were specific to training-induced improvements and not to baseline multitasking performance. Similarly, cortical volumes for the DLPFC region did not change from pretraining to posttraining for either group (relevant training, $t_{(49)}=0.12, p=0.90$; irrelevant training, $t_{(49)}=1.19, p=$ 0.24 ; paired $t$ test).

\section{Discussion}

Collectively, our findings, from a large participant cohort, provide a structural basis for individual differences in training-induced multitasking performance improvements in the prefrontal cortex. These

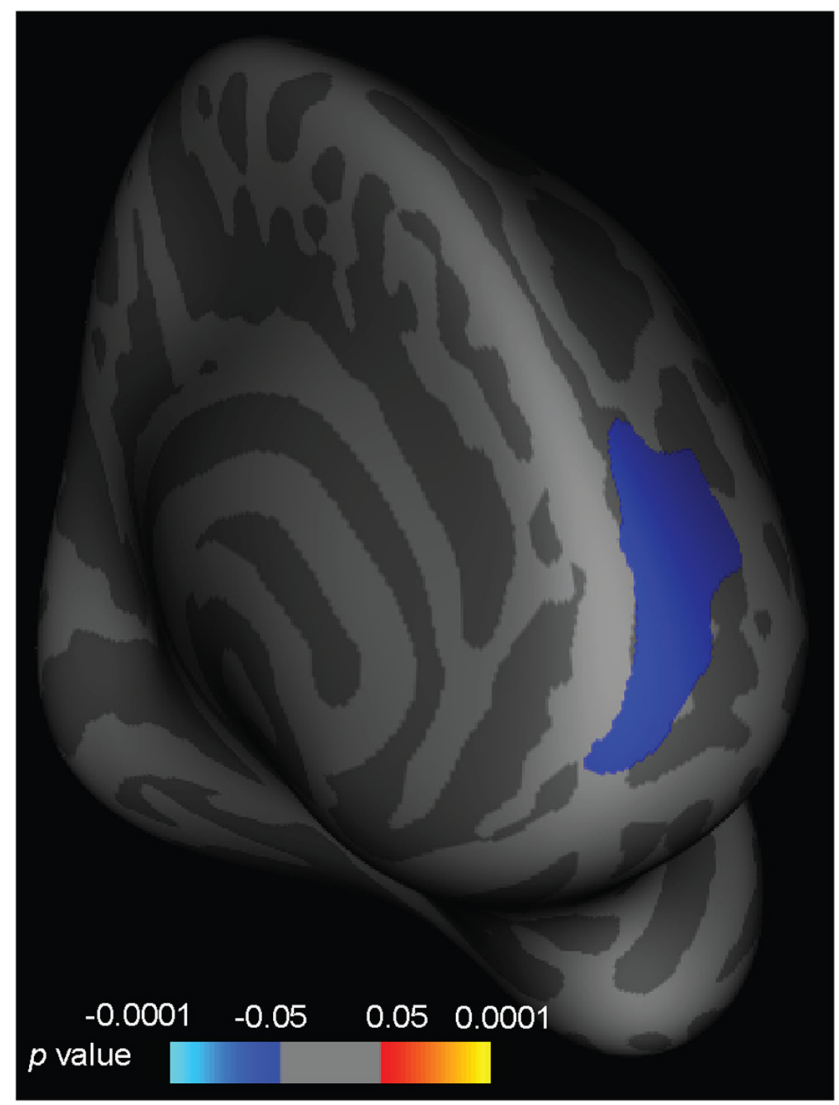

Figure 5. Correlations between structure and behavior for the multitask trials. The figure shows a whole-brain SBM analysis showing the correlation between volume and traininginduced performance gains for the multitask trials only (pretraining multitask trial RT minus posttraining multitask trial RT) in the relevant training group. The data depicted have been corrected for multiple comparisons at the $p<0.05$ level.

results are in agreement with a number of previous studies that implicated the lateral prefrontal cortex as a mediator of multitasking performance across a range of paradigms, such as a semantic judgment task combined with a spatial-rotation task (D'Esposito et al., 1995), color and letter discrimination dual tasks (Erickson et al., 2007), and even a clinical screening test (Tachibana et al., 2012). Most recently, a causal role was demonstrated for the left prefrontal cortex in improving multitasking performance in a gaming task using transcranial direct current stimulation (Hsu et al., 2015). Identification of the DLPFC (BA46) in our study ties in well with previous reports (Erickson et al., 2007) of increased functional activity after multitasking training in an anatomically adjacent DLPFC region (BA45; but see Dux et al., 2009 for other implicated regions). Although not the primary focus, complementary analysis from our multivoxel pattern analysis study (Garner and Dux, 2015) also showed that functional activity in the DLPFC demonstrated two key features that have been used previously as inclusive criteria when determining a brain region's involvement in multitasking behavior (Dux et al., 2006, 2009), namely, sensitivity to multitasking demands and increased activity to single tasks that is invariant to input and output modalities. Our current findings demonstrate that not only the function, but also the structure of the DLPFC determines the degree to which performance improvements can be accrued by multitasking training.

Our finding that smaller volumes within the left rostral DLPFC are associated with greater multitasking performance gains from a relevant training protocol departs from the classic "bigger is better" 
conception of brain structure-function relationships (de Sousa and Proulx, 2014). Instead, we demonstrate that individuals with smaller rostral DLPFC volumes have a propensity to gain more from multitasking training than those with larger volumes. Importantly, since the irrelevant training group showed no such associations with prefrontal cortex, these differences cannot be attributed to nontraining-related changes such as motivation, repetition, or other processes (Kelly and Garavan, 2005).

Bi et al. (2014) reported a similar inverse pattern of correlation between structure of the left fusiform face region and learning rate on a face perception task. They attributed their effect to cortical pruning mechanisms and speculated that inefficient synapses are pruned during development, resulting in smaller but more efficient cortical regions. However, an alternate explanation may be found in the underlying microanatomy of this region. For instance, a comparative neuroanatomical study undertaken at the microanatomical level (de Sousa and Proulx, 2014) reported that an increase in the volume of the primary visual cortex (V1) is associated with an increase in the number of neurons and decreased neuronal density. Since larger V1s are also associated with smaller anterior prefrontal cortices (Song et al., 2011), a systematic relationship may also exist between prefrontal volume and neuronal density. Exploring this relationship may be the key to understanding how information processing capacity is modulated by neural structure. Along a different vein, smaller DLPFC volumes have also been linked to greater blockto-block intraindividual variability in an executive episodic memory task (Lövdén et al., 2013), suggesting that training in these individuals might serve to reduce momentary lapses, boosting overall posttraining multitasking performance. In future work it will be important to disentangle the relationship between the macroanatomy and microanatomy of the prefrontal cortex and behavioral evaluation of trial-to-trial variability in multitasking performance.

Regardless of the precise mechanism, our finding that regional prefrontal volume is predictive of task-specific training improvements in the current dual-task paradigm is promising from a general perspective. Although the extent to which training benefits transfer to untrained tasks has not been conclusively established, there is some evidence for transfer of such dual-task training effects to paradigms with novel stimuli and response modalities (Liepelt et al., 2011; Lussier et al., 2012; Strobach et al., 2012; Anguera et al., 2013; Garner et al., 2014; Garner and Dux, 2015). Therefore, improvements on a multitasking training regimen may confer benefits that extend beyond the trained tasks. However, it remains to be ascertained whether underlying individual differences in brain structure also predict the degree to which performance improvements may transfer to untrained tasks. Regardless, the findings from this current work suggest that linking brain structure to behavior may be the key to unlocking the extent to which an individual's multitasking skills would improve after undergoing cognitive training using a multitasking training protocol. This could prove useful even from a clinical perspective when customizing health-care interventions for individuals with impairments in cognitive control, such as those with frontal lobe injuries. More broadly, our results highlight that cognitive potential is constrained by underlying brain structure. Elucidating the bounds of the structure-function relationship will be informative for understanding how and to what degree we can expand our cognitive potential.

\section{References}

Anguera JA, Boccanfuso J, Rintoul JL, Al-Hashimi O, Faraji F, Janowich J, Kong E, Larraburo Y, Rolle C, Johnston E, Gazzaley A (2013) Video game training enhances cognitive control in older adults. Nature 501:97101. CrossRef Medline
Bi T, Chen J, Zhou T, He Y, Fang F (2014) Function and structure of human left fusiform cortex are closely associated with perceptual learning of faces. Curr Biol 24:222-227. CrossRef Medline

Brainard DH (1997) The psychophysics toolbox. Spat Vis 10:433-436. CrossRef Medline

Chein JM, Schneider W (2005) Neuroimaging studies of practice-related change: fMRI and meta-analytic evidence of a domain-general control network for learning. Cogn Brain Res 25:607-623. CrossRef

Desikan RS, Ségonne F, Fischl B, Quinn BT, Dickerson BC, Blacker D, Buckner RL, Dale AM, Maguire RP, Hyman BT, Albert MS, Killiany RJ (2006) An automated labeling system for subdividing the human cerebral cortex on MRI scans into gyral based regions of interest. Neuroimage 31: 968-980. CrossRef Medline

Desimone R, Duncan J (1995) Neural mechanisms of selective visual attention. Ann Rev Neurosci 18:193-222. CrossRef Medline

de Sousa AA, Proulx MJ (2014) What can volumes reveal about human brain evolution? A framework for bridging behavioral, histometric, and volumetric perspectives. Front Neuroanat 8:51. Medline

D’Esposito M, Detre JA, Alsop DC, Shin RK, Atlas S, Grossman M (1995) The neural basis of the central executive system of working-memory. Nature 378:279-281. CrossRef Medline

Dux PE, Ivanoff J, Asplund CL, Marois R (2006) Isolation of a central bottleneck of information processing with time-resolved FMRI. Neuron 52: 1109-1120. CrossRef Medline

Dux PE, Tombu MN, Harrison S, Rogers BP, Tong F, Marois R (2009) Training improves multitasking performance by increasing the speed of information processing in human prefrontal cortex. Neuron 63:127-138. CrossRef Medline

Engvig A, Fjell AM, Westlye LT, Moberget T, Sundseth Ø, Larsen VA, Walhovd KB (2010) Effects of memory training on cortical thickness in the elderly. Neuroimage 52:1667-1676. CrossRef Medline

Erickson KI, Colcombe SJ, Wadhwa R, Bherer L, Peterson MS, Scalf PE, Kim JS, Alvarado M, Kramer AF (2007) Training-induced functional activation changes in dual-task processing: an FMRI study. Cerebral Cortex 17:192-204. Medline

Escorial S, Román FJ, Martínez K, Burgaleta M, Karama S, Colom R (2015) Sex differences in neocortical structure and cognitive performance: a surface-based morphometry study. Neuroimage 104:355-365. CrossRef Medline

Fischl B, Dale AM (2000) Measuring the thickness of the human cerebral cortex from magnetic resonance images. Proc Natl Acad Sci U S A 97: 11050-11055. CrossRef Medline

Fischl B, Sereno MI, Dale AM (1999) Cortical surface-based analysis. II: Inflation, flattening, and a surface-based coordinate system. Neuroimage 9:195-207. CrossRef Medline

Garner KG, Dux PE (2015) Training conquers multitasking costs by dividing task representations in the frontoparietal-subcortical system. Proc Natl Acad Sci U S A 112:14372-14377. CrossRef Medline

Garner KG, Tombu MN, Dux PE (2014) The influence of training on the attentional blink and psychological refractory period. Attention Percept Psychophys 76:979-999. CrossRef

Hayden EC (2012) Treating schizophrenia: game on. Nature 483:24-26. CrossRef Medline

Hsu WY, Zanto TP, Anguera JA, Lin YY, Gazzaley A (2015) Delayed enhancement of multitasking performance: effects of anodal transcranial direct current stimulation on the prefrontal cortex. Cortex 69:175-185. CrossRef Medline

Jaeggi SM, Buschkuehl M, Jonides J, Shah P (2011) Short- and long-term benefits of cognitive training. Proc Natl Acad Sci U S A 108:10081-10086. CrossRef Medline

Kamienkowski JE, Pashler H, Dehaene S, Sigman M (2011) Effects of practice on task architecture: combined evidence from interference experiments and random-walk models of decision making. Cognition 119: 81-95. CrossRef Medline

Kelly AM, Garavan H (2005) Human functional neuroimaging of brain changes associated with practice. Cereb Cortex 15:1089-1102. Medline

Liepelt R, Strobach T, Frensch P, Schubert T (2011) Improved intertask coordination after extensive dual-task practice. Quart J Exp Psychol 64: 1251-1272. CrossRef

Lövdén M, Schmiedek F, Kennedy KM, Rodrigue KM, Lindenberger U, Raz N (2013) Does variability in cognitive performance correlate with frontal brain volume? Neuroimage 64:209-215. CrossRef Medline 
Lussier M, Gagnon C, Bherer L (2012) An investigation of response and stimulus modality transfer effects after dual-task training in younger and older. Front Hum Neurosci 6:129. Medline

Martens HA, Dardenne P (1998) Validation and verification of regression in small data sets. Chemometr Intell Lab Syst 44:99-121. CrossRef

Owen AM, Hampshire A, Grahn JA, Stenton R, Dajani S, Burns AS, Howard RJ, Ballard CG (2010) Putting brain training to the test. Nature 465: 775-778. CrossRef Medline

Panizzon MS, Fennema-Notestine C, Eyler LT, Jernigan TL, Prom-Wormley E, Neale M, Jacobson K, Lyons MJ, Grant MD, Franz CE, Xian H, Tsuang M, Fischl B, Seidman L, Dale A, Kremen WS (2009) Distinct genetic influences on cortical surface area and cortical thickness. Cereb Cortex 19:2728-2735. CrossRef Medline

Pashler H (1994) Dual-task interference in simple tasks: data and theory. Psychol Bull 116:220-244. CrossRef Medline

Pelli DG (1997) The VideoToolbox software for visual psychophysics: transforming numbers into movies. Spat Vis 10:437-442. CrossRef Medline

Righart R, Duering M, Gonik M, Jouvent E, Reyes S, Hervé D, Chabriat H, Dichgans M (2013) Impact of regional cortical and subcortical changes on processing speed in cerebral small vessel disease. Neuroimage Clin 2:854-861. CrossRef Medline

Ruthruff E, Van Selst M, Johnston JC, Remington R (2006) How does prac- tice reduce dual-task interference: integration, automatization, or just stage-shortening? Psychol Res 70:125-142. CrossRef Medline

Song C, Schwarzkopf DS, Kanai R, Rees G (2011) Reciprocal anatomical relationship between primary sensory and prefrontal cortices in the human brain. J Neurosci 31:9472-9480. CrossRef Medline

Strobach T, Frensch PA, Soutschek A, Schubert T (2012) Investigation on the improvement and transfer of dual-task coordination skills. Psychol Res 76:794-811. CrossRef Medline

Strobach T, Liepelt R, Pashler H, Frensch PA, Schubert T (2013) Effects of extensive dual-task practice on processing stages in simultaneous choice tasks. Atten Percept Psychophys 75:900-920. CrossRef Medline

Tachibana A, Noah JA, Bronner S, Ono Y, Hirano Y, Niwa M, Watanabe K, Onozuka M (2012) Activation of dorsolateral prefrontal cortex in a dual neuropsychological screening test: an fMRI approach. Behav Brain Funct 8:26. CrossRef Medline

Van Selst M, Ruthruff E, Johnston JC (1999) Can practice eliminate the psychological refractory period effect? J Exp Psychol Human Percept Perform 25:1268-1283. CrossRef

Winkler AM, Kochunov P, Blangero J, Almasy L, Zilles K, Fox PT, Duggirala R, Glahn DC (2010) Cortical thickness or grey matter volume? The importance of selecting the phenotype for imaging genetics studies. Neuroimage 53:1135-1146. CrossRef Medline 